DRAFT VERSION OCTOBER 25, 2018

Preprint typeset using LTEX style emulateapj v. 04/20/08

\title{
PARTICLE CLUMPING AND PLANETESIMAL FORMATION DEPEND STRONGLY ON METALLICITY
}

\author{
ANDERS JOHANSEN \\ Leiden Observatory, Leiden University, P.O. Box 9513, 2300 RA Leiden, The Netherlands
}

\author{
ANDREW YOUDIN \\ Canadian Institute for Theoretical Astrophysics, University of Toronto, 60 St. George Street, Toronto, Ontario M5S 3H8, Canada \\ AND \\ MORDECAI-MARK MAC LOW \\ Department of Astrophysics, American Museum of Natural History, 79th Street at Central Park West, New York, NY 10024-5192, USA \\ Draft version October 25, 2018
}

\begin{abstract}
We present three-dimensional numerical simulations of particle clumping and planetesimal formation in protoplanetary disks with varying amounts of solid material. As centimeter-size pebbles settle to the midplane, turbulence develops through vertical shearing and streaming instabilities. We find that when the pebbleto-gas column density ratio is 0.01 , corresponding roughly to solar metallicity, clumping is weak, so the pebble density rarely exceeds the gas density. Doubling the column density ratio leads to a dramatic increase in clumping, with characteristic particle densities more than ten times the gas density and maximum densities reaching several thousand times the gas density. This is consistent with unstratified simulations of the streaming instability that show strong clumping in particle dominated flows. The clumps readily contract gravitationally into interacting planetesimals of order $100 \mathrm{~km}$ in radius. Our results suggest that the correlation between host star metallicity and exoplanets may reflect the early stages of planet formation. We further speculate that initially low metallicity disks can be particle enriched during the gas dispersal phase, leading to a late burst of planetesimal formation.

Subject headings: diffusion — hydrodynamics — instabilities — planetary systems: protoplanetary disks — solar system: formation — turbulence
\end{abstract}

\section{INTRODUCTION}

The concentration of particles to high spatial densities promotes the formation of planetesimals, the super-kilometer scale building blocks of planets. Drag forces on pebbles and rocks in disks lead to spontaneous particle clumping (Goodman \& Pindor 2000). The discovery of a linear streaming instability (Youdin \& Goodman 2005) shows that clumping is a robust consequence of particles drifting in and gas flowing out in disks with some radial pressure support (Nakagawa et al. 1986). Johansen \& Youdin (2007) studied the non-linear saturation of the streaming instability, neglecting vertical gravity and self-gravity. Those simulations showed that groups of boulders accelerate the gas around them towards the Keplerian velocity, reducing the radial drift locally and leading to temporary concentrations of boulders (see also Johansen et al. 2006).

Chiang (2008) and Barranco (2009) recently performed three-dimensional (3D) simulations of vertical shear instabilities in Keplerian disks in the single fluid limit where particles and gas have exactly the same velocities. These studies confirmed expectations that mid-plane turbulence develops when the Richardson number $\mathrm{Ri} \lesssim 1$. While perfect coupling is a good approximation for small grains, it cannot include vertical settling or in-plane streaming motions.

In this Letter we present 3D simulations of the motion of gas and pebbles in sub-Keplerian disks, including vertical gravity and particle sedimentation. Thus, we can study the combined effect of vertical shearing and streaming instabil-

Electronic address: ajohan@strw.leidenuniv.nl ities, as particles self-consistently settle towards - and are stirred from - the mid-plane. We exclude external sources of turbulence, including magnetorotational instabilities (which can actually promote clumping, see Johansen et al. 2007; Balsara et al. 2009). Our hydrodynamical simulations offer a first approximation to dead zones with low ionization (Sano et al. 2000) where turbulent surface layers drive only weak motions in the mid-plane (Fleming \& Stone 2003; Oishi et al. 2007).

In this non-magnetized limit, we investigate the clumping of smaller particles than considered in Johansen et al. (2007), which increases the likelihood of coagulation up to the initial sizes. We find that clumping of pebbles in the mid-plane layer increases sharply above a threshold mass fraction of solids roughly consistent with solar metallicity. Thus planetesimal formation may help explain the high probability of finding giant exoplanets around stars rich in heavy elements Gonzalez 1997; Santos et al. 2001; Fischer \& Valenti 2005; Johnson \& Apps 2009).

\section{SIMULATIONS}

We perform 3D hybrid simulations. They model gas on a fixed grid and solids with superparticles, each representing a swarm of actual particles. We solve the standard shearing sheet dynamical equations for a frame rotating at the Keplerian frequency $\Omega$ at a fixed orbital distance $r$ from the star. The axes are oriented such that $x$ points radially outwards, $y$ points in the orbital direction, while $z$ points vertically out of the disk. The gas is subject to a global radial pressure gradient that reduces the gas orbital speed by $\Delta v=-0.05 c_{\mathrm{s}} \approx 25 \mathrm{~m} \mathrm{~s}^{-1}$ 

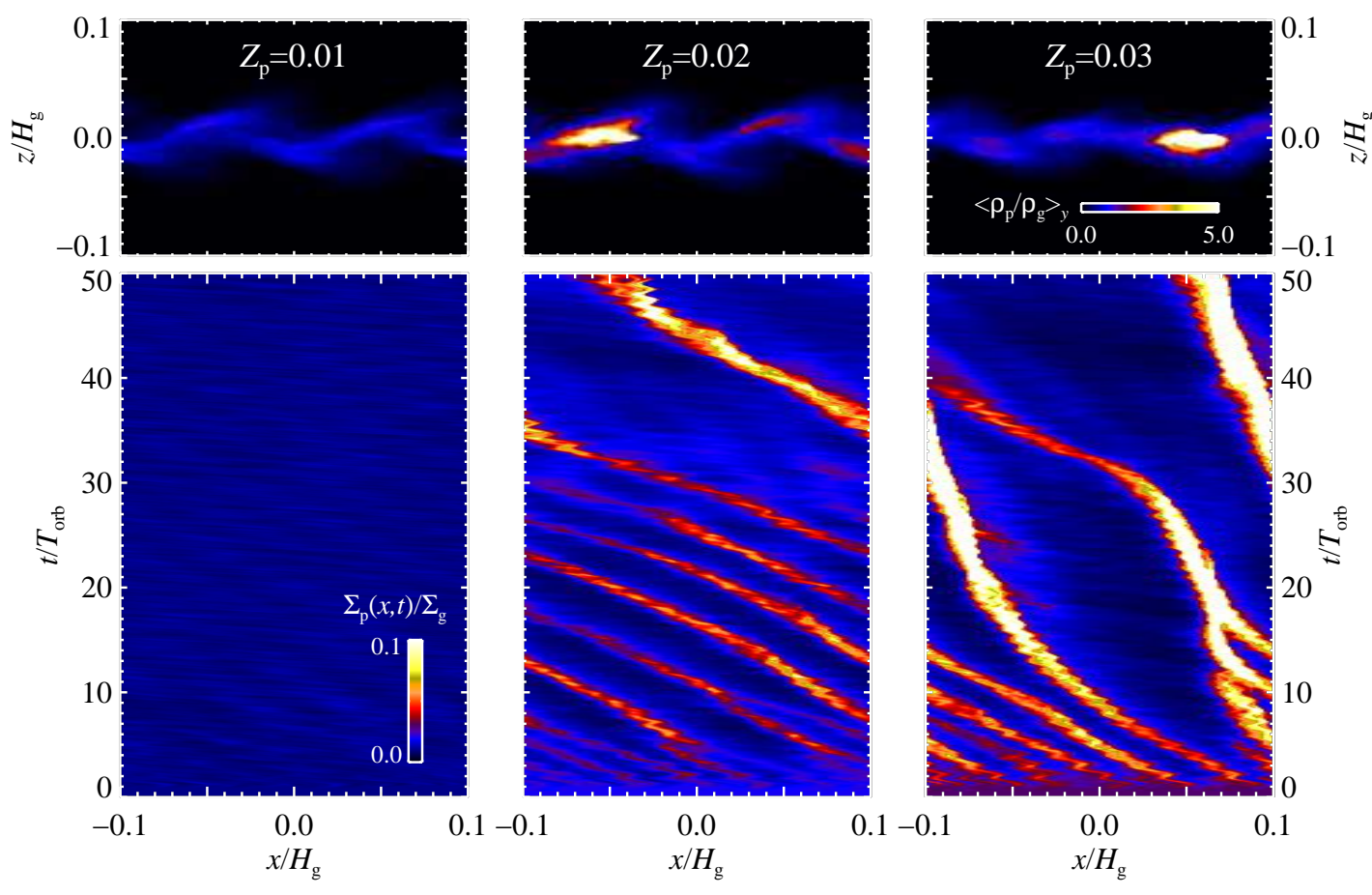

FIG. 1.- Results of $128^{3}$ sedimentation simulations for various values of the pebble abundance $Z_{\mathrm{p}}$ (different columns). The upper row of images shows the particle density averaged over the azimuthal direction at the end of each simulation. The wave-like pattern in the particle density is a consequence of coherent radial drift and vertical oscillations. The lower row shows particle density averaged over both $y$ and $z$ directions, as a function of radial coordinate $x$ and time $t$. At roughly solar metallicity (left column) the particle surface density is uniform. Super-solar metallicities (middle and right columns) produce significant clumps that merge over time because weaker clumps drift faster.

(Chiang \& Goldreich 1997). The sound speed $c_{\mathrm{s}}$, gas scale height $H_{\mathrm{g}}=c_{\mathrm{s}} / \Omega$ and mid-plane gas density $\rho_{0}$ are the natural units of the simulation.

The motion of gas and particles are coupled through momentum-conserving drag forces with particle friction time $\tau_{\mathrm{f}}$. Our dynamical equations are identical to those of Youdin \& Johansen (2007), with the addition of a vertical gravitational acceleration $g_{z}=-\Omega^{2} z$ affecting both gas and particles.

The superparticles are evenly distributed in mass and number into four bins of normalized friction time $\Omega \tau_{\mathrm{f}}=$ $0.1,0.2,0.3,0.4$. These friction times are characteristic of compact solids with radius $a_{\mathrm{p}} \approx 3,6,9,12 \mathrm{~cm}$ at $r=5 \mathrm{AU}$ in the Minimum Mass Solar Nebula (Weidenschilling 1977a b; Hayashi 1981). Rescaling to $r=10$ AU yields $a_{\mathrm{p}} \approx 1-4 \mathrm{~cm}$. We colloquially refer to this range of particle sizes as pebbles to contrast with larger $\Omega \tau_{\mathrm{f}} \approx 1.0$ boulders. The total pebble mass is fixed by setting the pebble-to-gas column density ratio $Z_{\mathrm{p}}=\left\langle\Sigma_{\mathrm{p}}\right\rangle /\left\langle\Sigma_{\mathrm{g}}\right\rangle$, where $\left\langle\Sigma_{\mathrm{p}}\right\rangle$ and $\left\langle\Sigma_{\mathrm{g}}\right\rangle$ are the mean particle and gas column densities, taking into account that most of the gas resides beyond the vertical extent of the simulation box. This pebble abundance turns out to be the crucial parameter for triggering particle clumping.

The total abundance of condensable materials beyond the ice line was estimated by Hayashi (1981) to be $Z_{\mathrm{c}} \approx 0.018$, while more up-to-date models give a somewhat lower value of $Z_{\mathrm{c}} \approx 0.015$ at temperatures less than $41 \mathrm{~K}$ (Lodders 2003; Allende Prieto et al. 2001). For our models a greater uncertainty is the efficiency of conversion from dust grains to pebbles. Assuming that a majority $(\approx 2 / 3)$ of the condensable solids are bound in pebbles, $Z_{\mathrm{p}}=0.01$ corresponds to solar metallicity. We also experiment with higher values of $Z_{\mathrm{p}}=0.02$ and $Z_{\mathrm{p}}=0.03$, which are motived both by stars with super-solar metallicities and by mechanisms that enrich the solids-to-gas ratio in disks (see \$6). A given pebble abundance would correspond to higher values of the metallicity if pebbles make up a smaller fraction of the condensable material.

We use a box size of $L_{x}=L_{y}=L_{z}=0.2 H_{\mathrm{g}}$ and resolutions of $64^{3}$ zones with 125,000 particles, and $128^{3}$ zones with $1,000,000$ particles. This relatively small box size is chosen to capture typical wavelengths of streaming and KelvinHelmholtz instabilities. The gas density is in vertical hydrostatic equilibrium. Particle positions are initialized to give a Gaussian density distribution around the mid-plane with scale height $H_{\mathrm{p}}=0.02 H_{\mathrm{g}}$, while gas and particle velocities are initially set to match the drag force equilibrium solution of Nakagawa et al. (1986).

\section{PARTICLE CLUMPING}

Since the disk is initially laminar, particles settle to the disk mid-plane. As particles collect in the mid-plane, they accelerate gas there towards the Keplerian orbital speed. This generates vertical shear that can drive Kelvin-Helmholtz instabilities. The velocity difference between gas and solids also triggers streaming instabilities. The resulting turbulence halts particle sedimentation and can lead to clumping.

In Fig. 1 we show results for pebble abundances $Z_{\mathrm{p}}=$ 0.01 (roughly solar), $Z_{\mathrm{p}}=0.02$ (super-solar) and $Z_{\mathrm{p}}=0.03$ (strongly super-solar). The $Z_{\mathrm{p}}=0.01$ simulation shows minimal clumping, but reveals instead a surprising interaction between settling and stirring. The particle layer is not centered uniformly on the mid-plane, as usually assumed. Instead the solids organize in a wave-like pattern (top left panel of Fig. 1) produced by coherent vertical oscillations combined with radial drift. We note that the streaming instabil- 


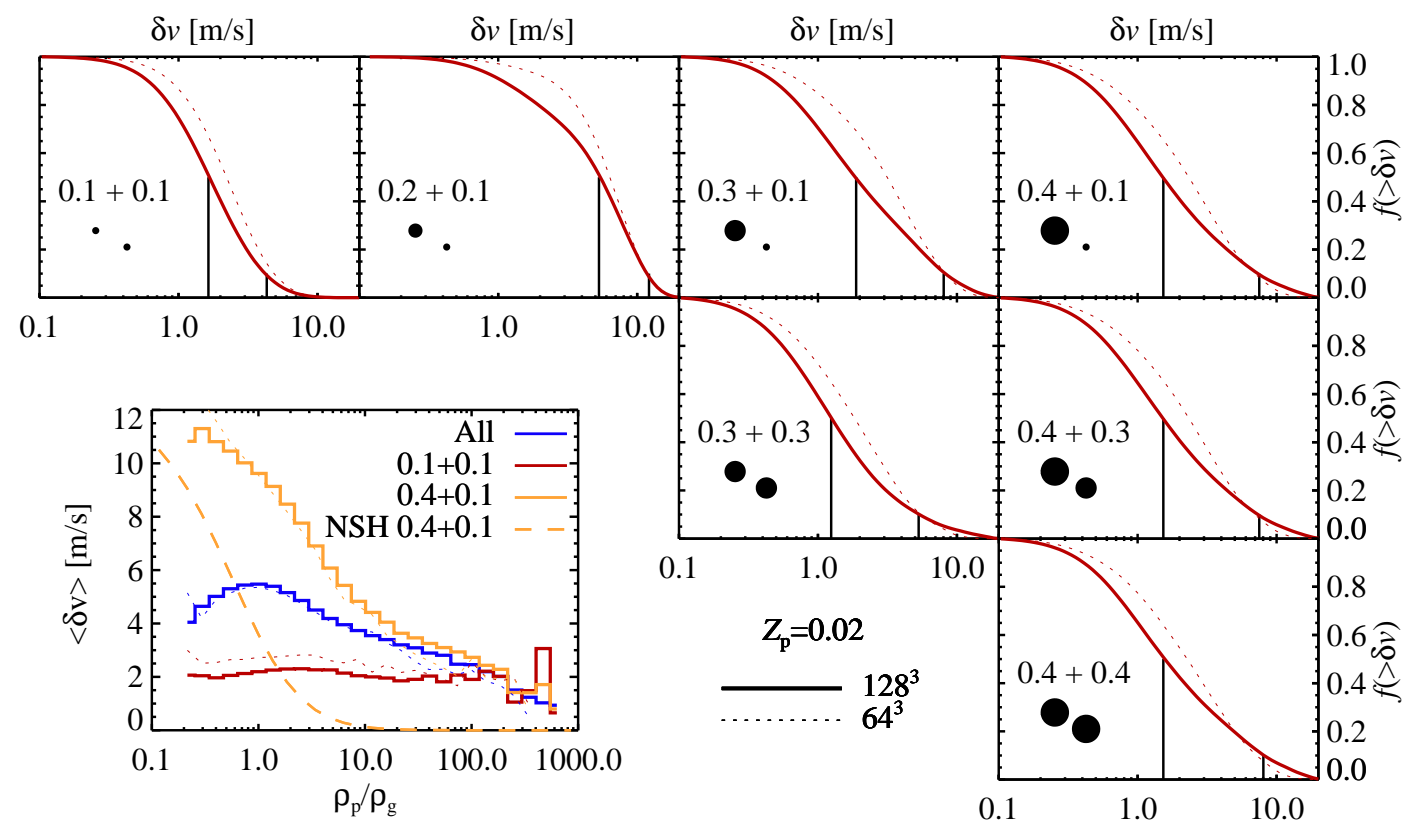

FIG. 2.- Cumulative distribution functions of the relative speed between particles of various sizes, weighted by the relative speed itself. Vertical lines mark $50 \%$ and $10 \%$ in the distribution functions. The typical collision speed lies between 0.5 and $5 \mathrm{~m} \mathrm{~s}^{-1}$, although collisions at up to $10 \mathrm{~m} \mathrm{~s}{ }^{-1}$ occur as well. Collision combinations $0.2+0.2,0.2+0.3,0.2+0.4$ showed very similar results and are not included in the figure. The low resolution simulation (dotted lines) shows a bit higher collision speeds, because less clumping occurs in it. The lower left panel shows the mean collision speed as a function of the density of particles in the grid cell. Grid cells with a high particle density have typical collision speeds below $2 \mathrm{~m} \mathrm{~s}^{-1}$. At low particle densities the collision speed between $\Omega \tau_{\mathrm{f}}=0.1$ and $\Omega \tau_{\mathrm{f}}=0.4$ particles rises, in agreement with differential drift (NSH analytical solution, Nakagawa et al. 1986), while the overall collision speed tends towards the collision speed between the $\Omega \tau_{\mathrm{f}}=0.1$ bodies that dominate low density regions.

ity is known to drive coherent vertical motions in clumps of boulders (Johansen \& Youdin 2007). The characteristic particle density $\tilde{\rho}_{\mathrm{p}} \equiv\left\langle\rho_{\mathrm{p}}^{2}\right\rangle /\left\langle\rho_{\mathrm{p}}\right\rangle \approx 0.9 \rho_{\mathrm{g}}$ is high enough to exert feedback on the gas. However the average mid-plane density $\rho_{\mathrm{p}}^{(\mathrm{mid})}=0.6 \rho_{\mathrm{g}}$ is diluted by the voids in the wave-like pattern, and is too small to trigger strong radial clumping.

Increasing the pebble abundance to $Z_{\mathrm{p}}=0.02$ and $Z_{\mathrm{p}}=0.03$ triggers strong overdensities in the particle layer (see Fig. 1). Initially, streaming instabilities produce many azimuthally extended clumps in the simulation box. Since the denser clumps drift inward more slowly, mergers result in a single dominant clump. The characteristic particle density increases due to the strong clumping, with $\tilde{\rho}_{\mathrm{p}} / \rho_{\mathrm{g}} \approx 0.9,18,73$ for $Z_{\mathrm{p}}=0.01,0.02,0.03$, respectively. The maximum density in the box increases from less than ten to more than 2,000 times the gas density when the pebble abundance is increased from $Z_{\mathrm{p}}=0.01$ to 0.02 .

So why does the clumping increase so sharply for order unity changes to the pebble abundance $Z_{\mathrm{p}}$ ? Fig. 11 (top panels) shows that the vertical extent of the wave-shaped particle layer decreases with $Z_{\mathrm{p}}$, because less kinetic energy is released in particle-dominated flows (Johansen \& Youdin 2007). Consequently the average mid-plane particle density increases, with $\rho_{\mathrm{p}}^{\text {(mid) }} / \rho_{\mathrm{g}}=0.6,2.0,9.0$ for $Z_{\mathrm{p}}=$ $0.01,0.02,0.03$. Strong clumping is thus expected for the higher metallicity cases, because the streaming instability is more powerful - for linear growth and nonlinear clumpingwhen the average $\rho_{\mathrm{p}} / \rho_{\mathrm{g}} \geq 1$ (Youdin \& Goodman 2005; Johansen \& Youdin 2007). A runaway develops as clumping limits stirring, giving higher average densities and in turn more clumping.

We can estimate the transitional value $Z_{\mathrm{p}}^{*}$ above which particles strongly clump. Assume that the sub-layer thickness is set by vertical shear turbulence to be $H_{\mathrm{p}} \sim \Delta v /(2 \Omega)$ as appropriate for small particles (Sekiya 1998; Youdin \& Shu 2002). Then the clumping threshold $\rho_{\mathrm{p}}^{\text {(mid) }} \gtrsim \rho_{\mathrm{g}}$ requires

$$
Z_{\mathrm{p}} \gtrsim Z_{\mathrm{p}}^{*}=\frac{H_{\mathrm{p}}}{H_{\mathrm{g}}}=\frac{\Delta v}{2 c_{\mathrm{s}}} .
$$

For $\Delta v=0.05 c_{\mathrm{s}}$ this yields a critical pebble abundance of $Z_{\mathrm{p}}^{*} \sim 0.025$. The slight overestimate of $Z_{\mathrm{p}}^{*}$ may arise because our pebbles settle more effectively than small grains. The result that clumping happens for lower $Z_{\mathrm{p}}$ when $\Delta v / c_{\mathrm{s}}$ is smaller (weaker radial pressure support) is seen in the simulations of Johansen et al. (2007).

The same metallicity threshold arises in particle layers with constant Richardson number (Sekiya 1998). Above $Z_{\mathrm{p}}^{*}$ a high density cusp forms in the mid-plane because the shear turbulence cannot lift solids with mass exceeding the local gas mass (Youdin \& Shu 2002). Vertical self-gravity causes the analytic cusps to diverge, while streaming instabilities are responsible for the strong clumping in our dynamical simulations.

\section{COLLISION SPEEDS}

The growth of solids into planetesimals depends on collision speeds, which affect the rates of fragmentation and coagulation (Blum \& Wurm 2008). The threshold speed for catastrophic destruction of a meter-scale body, with the material strength of compact rock, is around $10 \mathrm{~m} \mathrm{~s}^{-1}$ (Benz 2000). Porous aggregates, with less material strength, have an even lower threshold for destruction. Sticking of equal-sized bodies $\gtrsim 1 \mathrm{~mm}$ is not observed. However, small grains can adhere at speeds less than $\sim 1 \mathrm{~m} \mathrm{~s}^{-1}$ (Blum \& Wurm 2000).

From our simulation snapshots we measured relative speeds of all particle pairs within the same grid cell, giving 
$(1 / 2) N(N-1)$ unique collisions in a grid cell with $N$ particles. All collisions are assumed to be head on; for a random distribution of impact parameters the normal velocity would be reduced by $50 \%$ compared to the relative speed. We averaged over snapshots taken between 30 and 50 orbits of the $64^{3}$ and $128^{3}$ runs with $Z_{\mathrm{p}}=0.02$.

The distribution of collision speeds between particles of various sizes is shown in Fig. 2 We weight the occurrence of relative speeds by the speed itself to reflect that high speed collisions take place more frequently than low speed collisions. The typical collision speed lies in the interval 0.5$5 \mathrm{~m} \mathrm{~s}^{-1}$ for a sound speed $c_{\mathrm{s}} \approx 500 \mathrm{~m} \mathrm{~s}^{-1}$. Fig. 2 also shows that the collision speed is systematically lower in the denser particle clumps where the typical speed falls to less than $2 \mathrm{~m} \mathrm{~s}^{-1}$. Averaging collision speeds without weighting by the speed itself leads to much lower typical speeds, around 0.2 $2 \mathrm{~m} \mathrm{~s}^{-1}$.

The low collision speeds are surprising given the subKeplerian speed of $25 \mathrm{~m} \mathrm{~s}^{-1}$. The differential radial drift alone is $12.7 \mathrm{~m} \mathrm{~s}^{-1}$ between $\Omega \tau_{\mathrm{f}}=0.1$ and $\Omega \tau_{\mathrm{f}}=0.4$ test particles. However, the large bodies reside in dense clumps in the midplane layer where the differential radial drift is much reduced and the turbulence is weakened by mass loading (see insert in Fig. 2).

In simulations of particles in magnetorotational turbulence, collision speeds are measured to be much higher, of order $\sim 10 \mathrm{~m} \mathrm{~s}^{-1}$ (and well beyond for different-sized particles, see Johansen et al. 2007; Carballido et al. 2008). The more benign collision environment in the current simulations is more resistant to destruction and may even allow for growth by sticking. Low random velocities in high density clumps also favor gravitational collapse on a much shorter time-scale.

\section{SELF-GRAVITY}

Collective gravity can act to assemble solid particles into planetesimals (Safronov 1969; Goldreich \& Ward 1973; Youdin \& Shu 2002; Johansen et al. 2007; Cuzzi et al. 2008). The clumps in our $Z_{\mathrm{p}}=0.02$ and $Z_{\mathrm{p}}=0.03$ simulations reach densities beyond the Roche density,

$$
\rho_{\mathrm{R}}=\frac{9}{4 \pi} \frac{\Omega^{2}}{G} \sim 100 \rho_{\mathrm{g}},
$$

so self-gravity will be dynamically relevant. We activate the self-gravity in a $Z_{p}=0.02$ simulation that already developed for $\sim 40$ orbits, starting at a chosen time that displayed relatively weak clumping. A few orbits later a dense filament forms due to the streaming instability, but the concurrent action of the self-gravity leads to the condensation of seven gravitationally bound objects (see Fig. 3). Initially the planetesimals actively accrete pebbles from their surroundings. However, the accretion slows down after 5 orbits as $20 \%$ of the solid mass is locked in seven clumps with masses corresponding to solid bodies of radii between 100 and $200 \mathrm{~km}$. Further growth can occur both by accreting dust and pebbles and by planetesimal-planetesimal impacts, since the modest gas density fluctuations in mid-plane layer turbulence can not pump collision speeds of large planetesimals into the erosive regime (Ida et al. 2008; Y Yang et al. 2009).

\section{CONCLUSIONS}

In this Letter we present numerical evidence that the streaming instability, which arises in the coupled motion of gas and solids, can alone provide the necessary ingredients
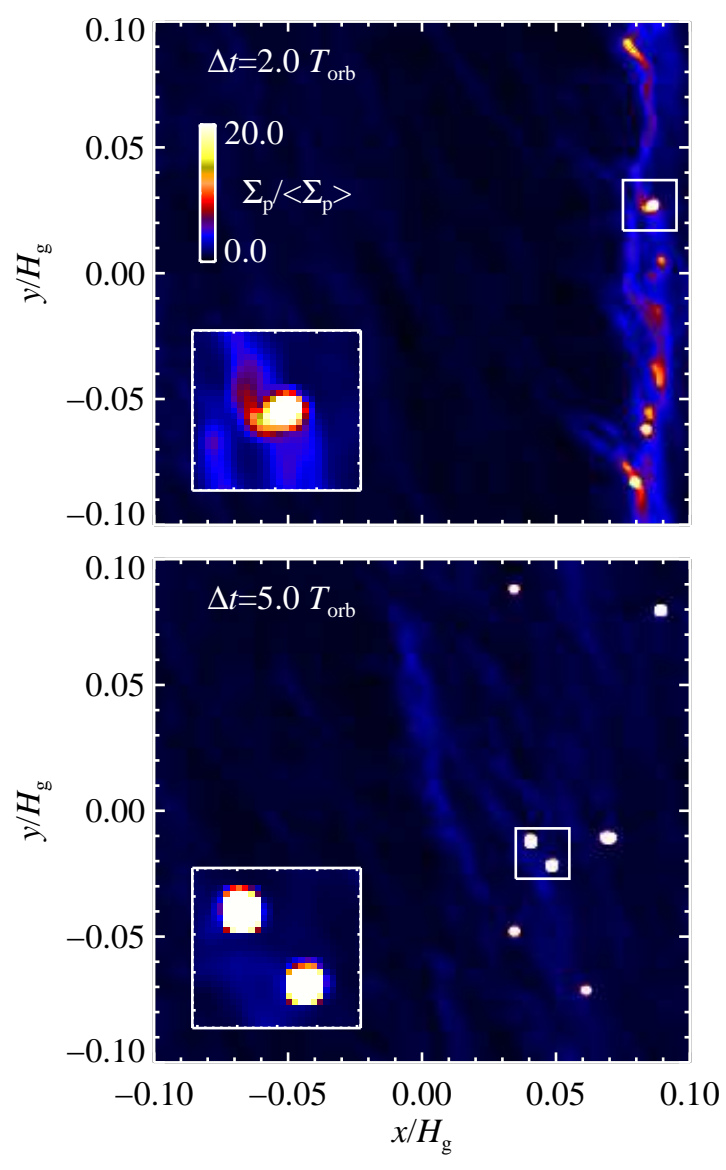

FIG. 3.- Snapshots of particle column density after self-gravity is activated. A dense nearly axisymmetric filament forms by the concurrent action of streaming instability and self-gravity, but it continues to fragment into gravitationally bound clusters of pebbles and rocks. The clusters initially show clear signs of active accretion of solids from the surroundings (top insert). After 5 orbits approximately $20 \%$ of the solid mass is in seven bound clusters, each containing a mass equivalent to a $100-200-\mathrm{km}$ scale solid body.

for the formation of planetesimals. With a high enough abundance of pebble-sized objects, the streaming instability drives the formation of high-density clumps in the sedimented midplane layer. Collision speeds inside those clumps are relatively low, around a few meters per second. The clumps get dense enough to contract gravitationally on a short time-scale into gravitationally bound clusters containing mass equivalent to solid planetesimals with radii of order $100 \mathrm{~km}$.

Our simulations display a threshold dependence of clumping on the global value of the pebble abundance. At a pebble abundance of $Z_{\mathrm{p}}=0.01$, corresponding roughly to solar metallicity, powerful turbulence develops and puffs up the mid-plane layer to such widths that clumping is strongly suppressed. This environment is not conducive to planetesimal growth, with low particle densities and high collision speeds. However, a doubling of the pebble abundance to $Z_{\mathrm{p}}=0.02$ leads to strong clumping with local peak particle densities exceeding a thousand times the gas density. This threshold behavior arises because the streaming instability triggers strong clumping when the particle density exceeds the gas density in the mid-plane.

If the fraction of condensables contained in pebbles is less than we assumed in this discussion (approximately 2/3), then the threshold we describe corresponds to higher values of the bulk metallicity. Coagulation-fragmentation mod- 
els (Brauer et al. 2008) and observations of pebbles in protoplanetary discs (Wilner et al. 2005) will be crucial for putting tighter constraints on the pebble abundance and the location of the metallicity switch.

The pebble abundance may increase as solid particles drift radially inward, converging in the inner few dozen AUs of the protoplanetary disk (Stepinski \& Valageas 1996; Youdin \& Chiang 2004). A higher $Z_{\mathrm{p}}$ also will occur if the gas in the disk preferentially evaporates. For example, photoevaporation removes gas from the disk surface, leaving behind larger solids that have sedimented to the mid-plane (Throop \& Bally 2005; Alexander \& Armitage 2007). This opens a window for late planet formation with planetesimals forming as the gas dissipates. The planets resulting from planetesimals formed so late will not have time enough to accrete a large gaseous envelope before the gas dissipates and would also suffer less severe type I migration (Youdin \& Shu 2002). This could explain why super-Earths and exo-Neptunes seem able to form equally well around stars of low and high metal- licity (Udry et al. 2006).

The correlation between host star metallicity and giant exoplanets can be partly attributed to the efficiency of forming several-Earth-mass cores from planetesimals (Ida \& Lin 2004; Mordasini et al. 2009). Our findings show that high metallicity already facilitates planetesimal formation, giving further support to the core accretion model for giant planet formation (Pollack et al. 1996).

Computer simulations were performed at the Huygens cluster in Amsterdam, funded by the NWO, and at the PIA cluster of the Max Planck Institute for Astronomy. AY and AJ are grateful to Chris Thompson for stimulating discussions at C.I.T.A. We thank the referee for careful reading of the manuscript. M-MML was partially supported by the NASA Origins of Solar Systems Program under grant NNX 07AI74G, and by the NSF Cyberenabled Discovery Initiative under grant AST08-35734.

\section{REFERENCES}

Alexander, R. D., \& Armitage, P. J. 2007, MNRAS, 375, 500

Allende Prieto, C., Lambert, D. L., \& Asplund, M. 2001, ApJ, 556, L63

Balsara, D. S., Tilley, D. A., Rettig, T., \& Brittain, S. A., 2009, MNRAS, 397,24

Barranco, J. A. 2009, ApJ, 691, 907

Benz, W. 2000, Space Science Reviews, 92, 279

Blum, J., \& Wurm, G. 2000, Icarus, 143, 138

Blum, J., \& Wurm, G. 2008, ARA\&A, 46,

Brauer, F., Dullemond, C. P., \& Henning, T. 2008, A\&A, 480, 859

Carballido, A., Stone, J. M., \& Turner, N. J. 2008, MNRAS, 386, 145

Chiang, E. I., \& Goldreich, P. 1997, ApJ, 490, 368

Chiang, E. 2008, ApJ, 675, 1549

Cuzzi, J. N., Hogan, R. C., \& Shariff, K. 2008, ApJ, 687, 1432

Fischer, D. A., \& Valenti, J. 2005, ApJ, 622, 1102

Fleming, T. \& Stone, J. M. 2003, ApJ, 585, 908

Goldreich, P., \& Ward, W. R. 1973, ApJ, 183, 1051

Gonzalez, G. 1997, MNRAS, 285, 403

Goodman, J., \& Pindor, B. 2000, Icarus, 148, 537

Hayashi, C. 1981, Prog. Theor. Phys., 70, 35

Ida, S., \& Lin, D. N. C. 2004, ApJ, 616, 567

Ida, S., Guillot, T., \& Morbidelli, A. 2008, ApJ, 686, 1292

Johansen, A., Henning, T., \& Klahr, H. 2006, ApJ, 643, 1219

Johansen, A., \& Youdin, A. 2007, ApJ, 662, 627

Johansen, A., Oishi, J. S., Mac Low, M.-M., Klahr, H., Henning, T., \& Youdin, A. 2007, Nature, 448, 1022

Johnson, J. A., \& Apps, K. 2009, ApJ, 699, 933

Lodders, K. 2003, ApJ, 591, 1220
Mordasini, C., Alibert, Y., Benz, W., \& Naef, D. 2009, A\&A, 501, 1161

Nakagawa, Y., Sekiya, M., \& Hayashi, C. 1986, Icarus, 67, 375

Oishi, J. S., Mac Low, M.-M., \& Menou, K. 2007, ApJ, 670, 805

Pollack, J. B., Hubickyj, O., Bodenheimer, P., Lissauer, J. J., Podolak, M., \& Greenzweig, Y. 1996, Icarus, 124, 62

Safronov, V. S. 1969, Evoliutsiia doplanetnogo oblaka. (English transl.: Evolution of the Protoplanetary Cloud and Formation of Earth and the Planets, NASA Tech. Transl. F-677, Jerusalem: Israel Sci. Transl. 1972)

Sano, T., Miyama, S. M., Umebayashi, T., \& Nakano, T. 2000, ApJ, 543, 486

Santos, N. C., Israelian, G., \& Mayor, M. 2001, A\&A, 373, 1019

Sekiya, M. 1998, Icarus, 133, 298

Stepinski, T. F., \& Valageas, P. 1996, A\&A, 309, 301

Throop, H. B., \& Bally, J. 2005, ApJ, 623, L149

Udry, S., Mayor, M., Benz, W., Bertaux, J.-L., Bouchy, F., Lovis, C.,

Mordasini, C., Pepe, F., Queloz, D., \& Sivan, J.-P. 2006, A\&A, 447, 361

Weidenschilling, S. J. 1977a, MNRAS, 180, 57

Weidenschilling, S. J. 1977b, Ap\&SS, 51, 153

Wilner, D. J., D’Alessio, P., Calvet, N., Claussen, M. J., \& Hartmann, L. 2005, ApJ, 626, L109

Yang, C.-C., Mac Low, M.-M., \& Menou, K. 2009, ApJ, submitted (arXiv:0907.1897)

Youdin, A. N., \& Shu, F. H. 2002, ApJ, 580, 494

Youdin, A. N., \& Chiang, E. I. 2004, ApJ, 601, 1109

Youdin, A. N., \& Goodman, J. 2005, ApJ, 620, 459

Youdin, A., \& Johansen, A. 2007, ApJ, 662, 613 